\title{
Performance Measurements of a New Large-Area Neutron Scintillation Detector System
}

\author{
G. Kemmerling, U. Bünten, U. Clemens, R. Engels, Member, IEEE, M. Heiderich, W. Pykhout-Hintzen, H. Rongen, \\ J. Schelten, D. Schwahn, and K. Zwoll
}

\begin{abstract}
During the upgrade of the small-angle neutron scattering instrument KWS-1 at the research reactor FRJ-2, the 15-yr-old detector system has been replaced. While the Anger camera-based concept of the detector remained unchanged, the signal and data processing branch has been completely revised. Because of higher count rate requirements, a highly configurable parallel readout electronics has been developed aiming at counting rates of several hundred kilohertz. The whole detector system has been recently installed at KWS-1 and measurements to study the system performance have been carried out. The linearity and spatial resolution of the new detector have been determined by an analysis of an acquired image taken with a hole diaphragm in front of the scintillator. The overall system dead time has been measured by comparing the detector count rates at several scattering intensities to the count rates achieved with a fission chamber. Finally, scattering patterns of well-known samples have been taken in order to demonstrate the quality of the acquired images.
\end{abstract}

Index Terms-Anger camera, position-sensitive scintillation neutron detector.

\section{INTRODUCTION}

$\mathbf{I}$ $\mathrm{N}$ small-angle neutron scattering (SANS), the structural and dynamical properties of condensed matter are analyzed [1]. Because of the low momentum transfer associated with scattering at small angles, relatively large objects may be investigated. Typical samples are, for instance, macromolecules and colloidal solutions with structure sizes in the range of 5 to 5000 $\AA$. To measure the total scattering regime, instruments for SANS measurements are usually equipped with large-area positionsensitive detectors. This allows for an efficient exploitation of the expensive beam-time.

The SANS-diffractometer KWS-1 at the research reactor FRJ-2 of the Forschungszentrum Jülich is such an instrument. It is based on the pinhole-technique with a monochromator and several collimators for wavelength selection and beam adjustment. At KWS-1, a position-sensitive detector [2] was successfully used for about 15 yr. However, after an installation of advanced neutron guides, there were higher intensities available leading to high dead times of the detector

Manuscript received November 29, 2003; revised March 11, 2004.

G. Kemmerling, U. Clemens, R. Engels, H. Rongen, and K. Zwoll are with the Zentrallabor für Elektronik, Forschungszentrum Jülich, D-52425 Jülich, Germany (e-mail: G.Kemmerling @fz-juelich.de).

M. Heiderich, U. Bünten, W. Pykhout-Hintzen, and D. Schwahn are with the Institut für Festkörperforschung, Forschungszentrum Jülich, D-52425 Jülich, Germany.

J. Schelten is with the Institut für Schichten- and Grenzflächen,

Forschungszentrum Jülich, D-52425 Jülich, Germany.

Digital Object Identifier 10.1109/TNS.2004.829576 at counting rates above $10 \mathrm{kHz}$. The limiting factor was the readout electronics. Due to an analog-based calculation of the neutron position, large shaping times of the analog signals were required, resulting in a dead time of about $10 \mu \mathrm{s}$. Thus, to take advantage of the higher neutron intensities, as well as to guarantee a long-term maintenance, the whole instrument has been upgraded. Within this upgrade, a new position-sensitive scintillation detector with a completely revised electronics branch has been installed. The new design comprises fast signal and data processing using modern technologies like field programmable-gate arrays (FPGAs) and digital signal processors (DSPs).

\section{Detector And Readout Electronics}

This section gives a short description of the concept of the new detector and readout electronics. It has already been described in a previous paper [3].

\section{A. Detector Principle and Front End}

Apart from a few technical innovations, the construction of the new detector is basically identical to the former detector and based on the principle of an Anger camera [4]. Thus, most design parameters were taken from a numerical simulation [5], which was carried out during the development of the old detector.

There is a 1-mm-thick ${ }^{6} \mathrm{Li}$-glass scintillator for the neutron capture with an efficiency of $96 \%$ for $8 \AA$ neutrons. It consists of $4 \times 4$ plates of dimensions $15 \mathrm{~cm} \times 15 \mathrm{~cm}$, which are glued with optical contact on a support glass frame, forming an active area of $60 \times 60 \mathrm{~cm}^{2}$. To increase the fraction of the collected photons, the scintillator is covered on the top side with reflecting paint. Behind the scintillator, there is a disperser glass in optical contact to an $8 \times 8$ array of photomultiplier tubes (PMTs) with 3 -in diameter, which are used for light detection. Because there is an air gap between scintillator and light disperser, a critical angle beyond which light undergoes total internal reflection is produced. Thus, the light received by the PMTs is limited to a cone, whose diameter on the PMT-plane depends linearly on the disperser thickness. As the simulation shows, this thickness influences the linearity and resolution of the detector. With increasing thickness the linearity improves while the resolution becomes worse. The optimal thickness of the disperser is $10 \%$ less than the separation of adjacent PMTs $(84 \mathrm{~mm})$, resulting in a disperser thickness of $75 \mathrm{~mm}$. Then the light cone produced by a neutron event covers an array of 9 PMTs. 


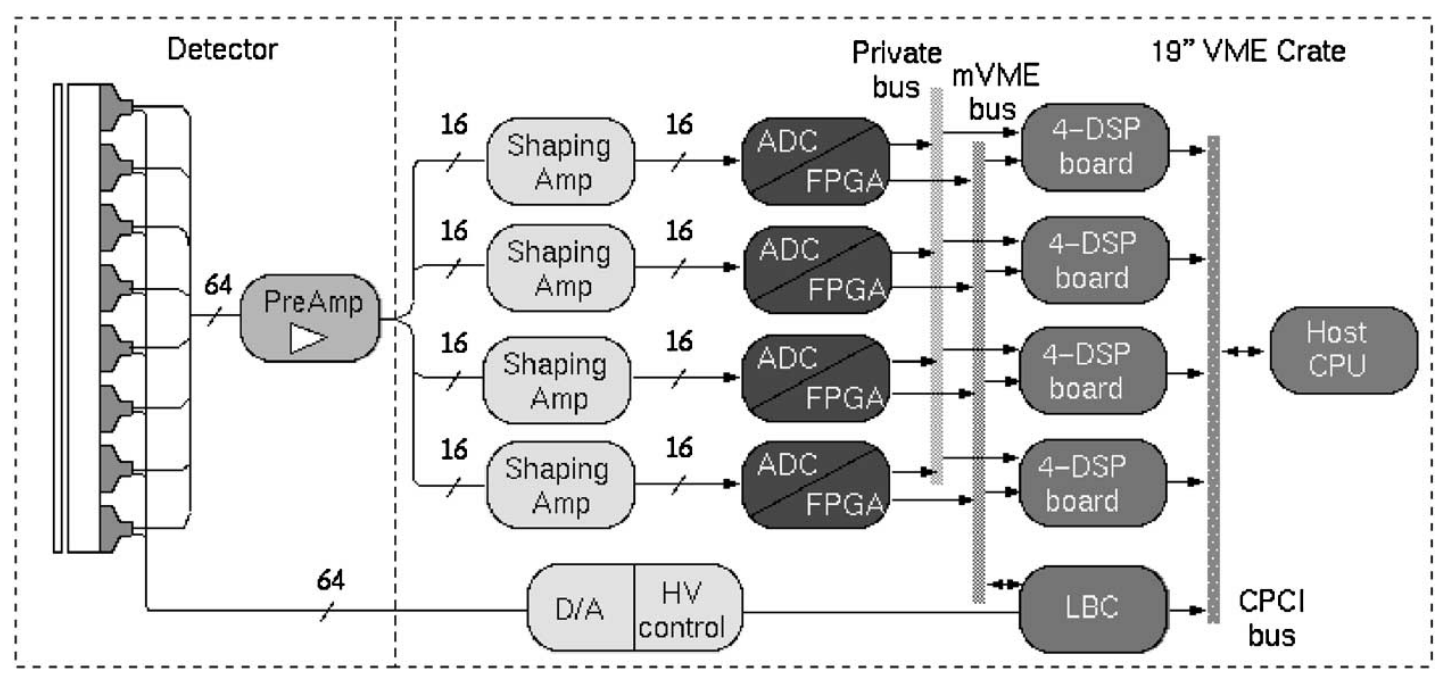

Fig. 1. Block diagramm of the analog and digital signal processing.

\section{B. Readout Electronics}

The electronics design is shown in Fig. 1. The mechanical assembly utilizes a single $6 \mathrm{U} 19^{\prime \prime}$ VME-crate, which has been equipped with three partly overlapping bus systems on the backplane. There is a private bus with CPCI connectors, used for the transfer of analog signals and board synchronization, a modified VME bus (mVME) for a fast data transfer of up to $80 \mathrm{MBytes} / \mathrm{s}$, and a standard CompactPCI bus (CPCI) for the communication with a host CPU. Four board types have been implemented according to the different steps in signal and data processing.

Detector signals are first amplified by preamplifiers. Each of the anodes of the 64 PMTs is connected to its preamplifier which is mounted close to the detector. The signals are then input to the analog board, which filters and amplifies the preamplifier signals. An analog board contains the main shaping amplifiers for 16 PMT channels. To achieve a sufficient signal-to-noise ratio (SNR), the PMT signals are differentiated and integrated to generate an amplified unipolar Gaussian pulse shape with a time constant of $200 \mathrm{~ns}$.

Signals are then fed to the analog-to-digital converter (ADC)/FPGA board, which has the task of signal digitization and event triggering. Because there are four boards of this type in the system, their clocks have to be synchronized to avoid electronic time displacements. This is done via the private bus. An ADC/FPGA board contains free running 10-bit ADCs for 16 channels and a FPGA (XILINX XCV300-BG432) with a local FIFO of 4096 36-bit words for data buffering. During measurements, the ADCs continuously digitize the signals with a clock rate of $20 \mathrm{MHz}$ and deliver their values to the FPGA. Then the FPGA performs a parallel peak detection on the basis of a sliding mean value determination [6], where a detected peak means a valid event.

The FPGAs are also responsible for a first step in background suppression. There is usually a large $\gamma$-background, originating directly from the reactor or from activated material in the surroundings. It has an energy spectrum in the $\mathrm{MeV}$ range with a strong intensity increase at lower energies. Particulary for measurements with weakly scattering samples, this background spoils the results, because of the sensitivity of the ${ }^{6} \mathrm{Li}$-scintillator for this radiation. Therefore, discriminators have been implemented inside the FPGA. By checking the crossing of lower levels at the beginning of the pulse and the falling below such a level at the end of the pulse, they should largely reduce noise and lower energy $\gamma \mathrm{s}$, as well as reject events with pile-up effects. Further background suppression is done during event reconstruction. By calculating the pulse height sum of the 9 PMTs, covered by the light cone, one can accurately determine events with pulse heights outside the range of the neutron peak. Thus, in the very first step of reconstruction one can filter out those events that do not belong to the neutron peak, which leads to a $\gamma$-ratio of order $10^{-4}$ per neutron.

Once a peak has been recognized, a signal is emitted to the mVME bus on which all FPGAs on different boards write their current values into the FIFO. Up to 800 events can be buffered in the FIFOs, which allows for an averaging of load distribution. For further data processing, two types of DSP-boards with mixed VME and CompactPCI interfaces are used. The first type, the so-called local bus controller (LBC), contains a single DSP (TMS320 C6201), which controls the data flow. On the second board type, there are four floating-point DSPs (TMS320 C6701), which are to be used for the reconstruction of the event position. Up to four such boards may be inserted in the system to provide sufficient computing power for position reconstruction. In case of a valid event, the LBC gets the signal from the corresponding FPGA, looks up a free DSP and instructs it to read the data out from the FPGA FIFO-buffers via the modified VME bus. There, the position of the event is calculated and the associated histogramming memory position is incremented. Accumulated data may then be accessed from the host CPU.

As it is known that the gain of PMTs may drift in time with a rate in the order of percents per week, a stabilization unit is also foreseen for the system. It utilizes a gain adjustment by changing the PMT high voltage with a digital-to-analog converter. So, by taking the pulse height spectra for each PMT, one can obtain a matching of average pulse heights of all PMTs by changing their high voltages. This is typically done once a week. 


\section{Position Reconstruction}

Position reconstruction is carried out by a two step process on the DSP-boards. At first, the coarse address of the event is determined by finding the photomultiplier with the highest signal amount and its corresponding $3 \times 3$ box. Inside this PMT with index $i$, the $\mathrm{x}$ and $\mathrm{y}$ positions are calculated according to the left/right light asymmetry of neighboring column and row sums, respectively

$$
q_{i}=\frac{S_{i+1}-S_{i-1}}{S_{i-1}+S_{i}+S_{i+1}} .
$$

In this equation, $S_{i}$ denotes the signal sum of the three PMTs at column/row $i$, that are covered by the light cone. The $q_{i}$ are monotonic functions of the position coordinates $\mathrm{x}$ and $\mathrm{y}$, respectively. In general, the functions are nonlinear. However, if the disperser thickness is properly chosen, and the photocathodes are uniform, the functions are almost linear. Their nonlinearity leads to intensity nonuniformities if the detector is uniformly illuminated with neutrons. There are also other intensity inhomogeneities due to light losses at the edges of the scintillator plates, local inhomogeneities of the light yield of the scintillator, and different coatings of the scintillator with the reflective paint. These are not tolerable for a physical analysis of the obtained images. To correct these shortcomings, a calibration measurement with an uniform neutron illumination of the detector must be performed. The inverse of the individual intensities of each channel, obtained by this method, acts as a correction factor for a proper sensitivity calibration of measured samples.

\section{RESULTS}

In November 2002, the former detector system was replaced by the new detector and readout electronics. After the start-up phase, measurements under neutron irradiation were carried out to determine and optimize the properties of the new detector system. This section reports on the results of these first measurements.

\section{A. Linearity and Position Resolution}

To determine the linearity and position resolution of the new detector, an experimental setup with an absorbing hole diaphragm in front of the scintillator was used. The diaphragm was boron coated with holes of $5 \mathrm{~mm}$ diameter and $5 \mathrm{~cm}$ spacings. Under irradiation, scattered neutrons are only measured at the hole positions and by an analysis of the peak profiles, the desired values are obtained. Fig. 2 shows the measured intensity distribution.

The integral linearity is determined from the variance $\sigma$ of the deviations of the peak positions $Q_{s}$ from their nominal values, whereas $s$ denotes either $\mathrm{x}$ or $\mathrm{y}$. With the peak positions derived from fitting Gaussian to the profiles and the nominal values calculated through a linear least-square fit $A \cdot s+B$ over all peaks in a row or column, a value of about $2.8 \%$ has been achieved for the integral linearity. It is the signal processing of the coarse fine addressing of the neutron capture events that guarantees this excellent integral linearity.

The differential linearity is determined by the ratio of the distance of adjacent peak positions to the distance of nominal peak

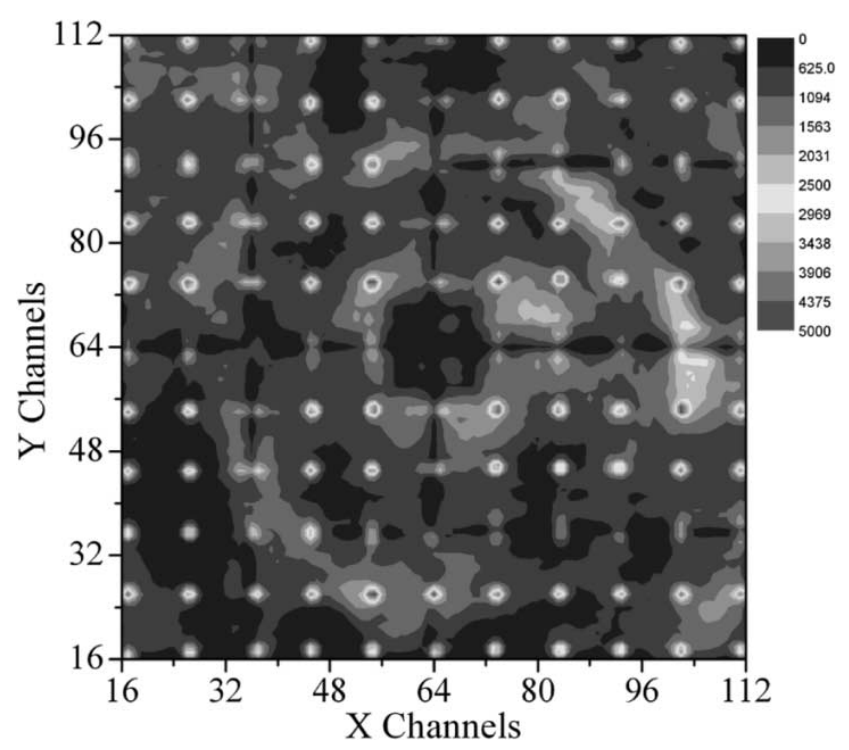

Fig. 2. Reconstructed image of hole diaphragm. The disappearance of some peaks is caused by their location just above the edges of the scintillator plates.

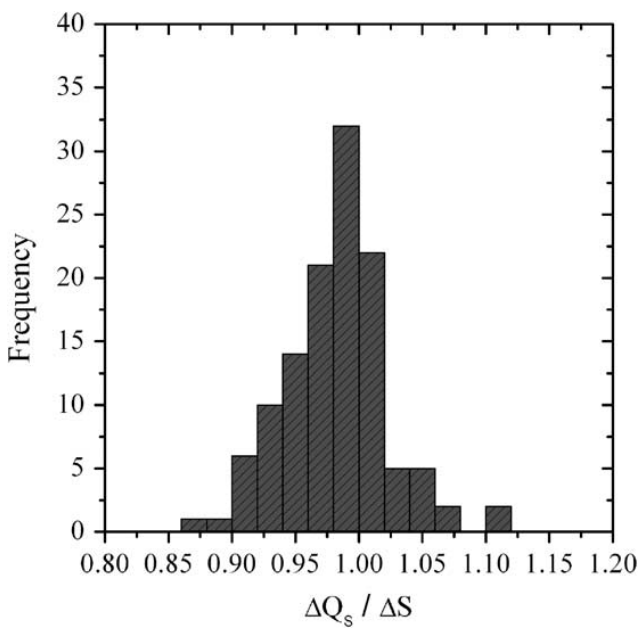

Fig. 3. Differential linearity of the detector. The histogram contains all derived values in $\mathrm{x}$ and $\mathrm{y}$ direction.

positions $\Delta Q_{s} / \Delta S=Q(s)$ and expressed by the maximum deviation from its mean value over all peaks $\bar{Q}(s): \max \mid Q(s)-$ $\bar{Q}(s) \mid$. Fig. 3 shows the values obtained for the new detector.

As with the old detector, the differential linearity is poor and its measured value is $12 \pm 0.5 \%$. It is caused by the incomplete matching of the fine/coarse addressing and leads to nonuniformities in the intensity distribution. However, since the detector response is stable due to the implemented gain stabilization, one is able to correct for these nonlinearities.

The spatial resolution of the detector depends on the total number of photoelectrons per capture event, on the separation and diameter of the PMTs, on the disperser thickness and the refractive indices of the scintillator and disperser, and on the local and global photoelectron conversion factors of the involved photocathodes. These influences have been investigated in numerical simulations [5], resulting in an estimated value of $\sigma \simeq 8.4$ $\mathrm{mm}$ for this detector.

Here, the position resolution is determined by the full-width at half-maximum (FWHM) of the fitted Gaussian at the peak 


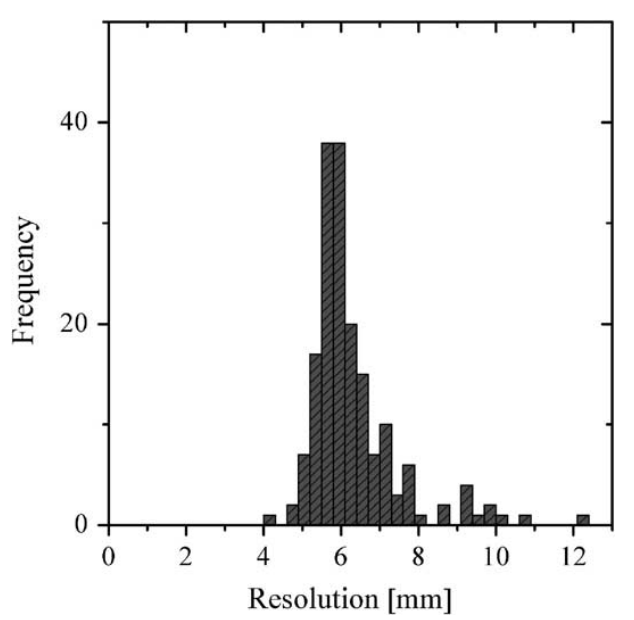

Fig. 4. Spatial resolution of the detector. The histogram contains the calculated values from all peaks.

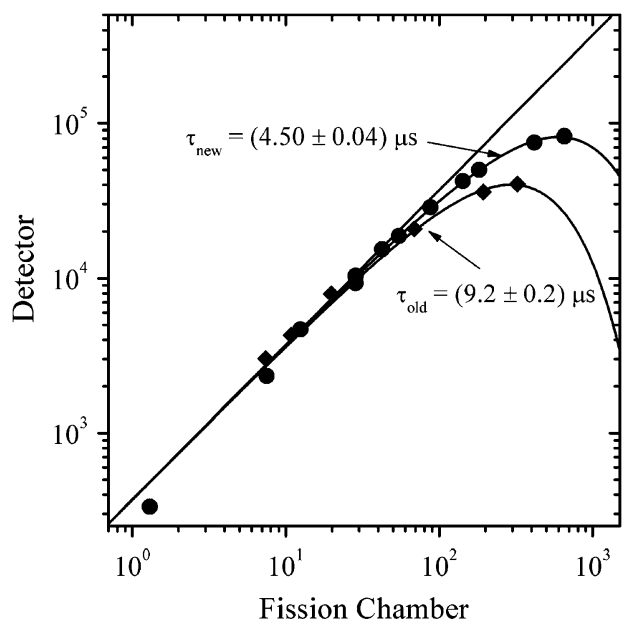

Fig. 5. Dead time measurements. The image shows the measured counting rates of the old and new detector versus that of a fission chamber.

positions $P_{\mathrm{FWHM}}$ and the hole diameter $H_{D}$. With these values, the FWHM of the spatial resolution $\sigma$ can be calculated by

$$
\sigma=\sqrt{P_{\mathrm{FWHM}}^{2}-H_{D}^{2}}
$$

Fig. 4 shows a histogram of the values obtained for the spatial resolution from all peaks. Because of the different light yield at the corresponding positions, the values vary from 4 to $11 \mathrm{~mm}$. The spatial resolution expressed by its mean value is $\sigma=6.2 \pm$ $1.2 \mathrm{~mm}$. This value is well adapted to SANS experiments, i.e., resolution corrections need not be applied.

\section{B. Dead Time Measurements}

The determination of the system dead time was carried out by comparing the detector counting rates under uniform neutron illumination to that of a fission chamber. Because the count rate of the fission chamber is linearly related to the count rate of the scattered neutrons, the dead time of the detector may be derived by the deviations of the count rates from ideal behavior.

Fig. 5 shows the counting rates measured with the old and new detector in relation to the counting rates of the fission chamber. Due to the pulse processing inside the FPGAs, the appropriate model to describe the dead time behavior of this detector is the

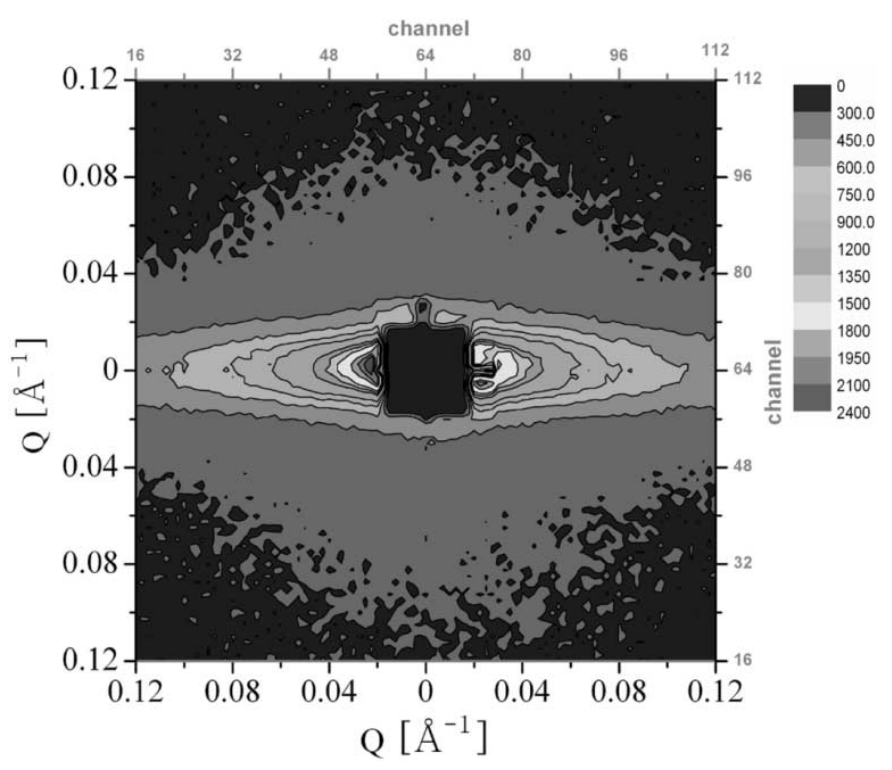

Fig. 6. Reconstructed and sensitivity corrected image. The image shows a measurement with a stretched polypropylene sample. Stretching was performed in vertical direction.

paralyzable dead time model, in which at high counting rates the dead period of the detector may be multiply extended. In this model the measured counting rate $\mathrm{m}$ of the detector is obtained by the true rate $n$ through

$$
m=n \cdot e^{-n \tau}
$$

where $\tau$ denotes the dead time. By curve fitting a value of $\tau=$ $4.5 \pm 0.5 \mu \mathrm{s}$ is obtained for the new detector, which is only about half of the dead time of the former detector.

\section{Image Quality}

To demonstrate the quality of the acquired images, scattering patterns were measured with samples which were previously investigated with the former detector. Fig. 6 shows a reconstructed and sensitivity corrected image of an anisotropic scatterer that is well suited to demonstrate the image quality. It represents a stretched polypropylene sample, where the scattering centers are polymer molecules.

One immediately sees the twofold symmetry of the scattering pattern with a strong and weak intensity decay parallel and perpendicular to the stretching direction. There are no deformations or additional detector-induced substructures observable, indicating that the position reconstruction as well as the sensitivity correction work reliably.

\section{CONCLUSION}

The performance of a new position-sensitive detector system for small-angle neutron scattering experiments has been investigated. First measurements were carried out by using an absorbing hole diaphragm in front of the detector. By analyzing the spacing of the peak positions, integral and differential linearity have been determined to be $2.8 \%$ and $12 \%$, respectively. These values are in good agreement with simulations and are slightly better than for the former detector. 
The spatial resolution of the detector is derived from the width of the peaks. It varies due to position-dependant inhomogeneities of the light yield. The spatial resolution expressed by its mean value is $\sigma=6.2 \mathrm{~mm}$, which is well adapted to SANS experiments. The dead time of the detector system has been determined with a uniform neutron illumination. By comparing the counting rates of the detector and a fission chamber, a dead time of $4.5 \mu$ s has been achieved. This is already a considerable improvement relative to the former detector system, but there is also a potential for a further reduction of this value by an optimization of data transfer in the readout electronics.

Finally, image quality was demonstrated by measuring the scattering pattern of an anisotropic scatterer. For such scatterers, large-area position-sensitive detectors are necessary. There were no deformations or detector-induced substructures visible, demonstrating that the position reconstruction and the applied sensitivity corrections work reliably.

\section{REFERENCES}

[1] J. Schelten and R. W. Hendricks, "Recent developments in X-ray and neutron small-angle scattering instrumentation and data analysis," $J$. Appl. Cryst., vol. 11, pp. 297-324, 1978.

[2] M. Heiderich, R. Reinartz, R. Kurz, and J. Schelten, "A two dimensional scintillation detector for small angle neutron scattering," Nucl. Instrum. Methods, vol. A 305, pp. 423-432, 1991.

[3] G. Kemmerling, N. Bussmann, U. Clemens, R. Engels, M. Heiderich, R. Reinartz, H. Rongen, J. Schelten, D. Schwahn, and K. Zwoll, "A new two-dimensional scintillation detector for small angle neutron scattering experiments," IEEE Trans. Nucl. Sci., vol. 48, pp. 1114-1117, 2001.

[4] H. O. Anger, "Scintillation camera," Rev. Sci. Instr., vol. 29, pp. 27-33, 1958.

[5] J. Schelten and R. Kurz, "Computer modeling of position-sensitive scintillation detectors," in Proc. Conf. Position-Sensitive Detection of Thermal Neutrons, P. Convert and J. B. Forsyth, Eds. London, U.K., 1983 , p. 203

[6] P. J. Pietraski, Z. Zojceski, D. P. Siddons, G. C. Smith, and B. Yu, "Digital centroid-finding electronics for high-rate detectors," IEEE Trans. Nucl. Sci., vol. 46, pp. 810-816, 1999. 